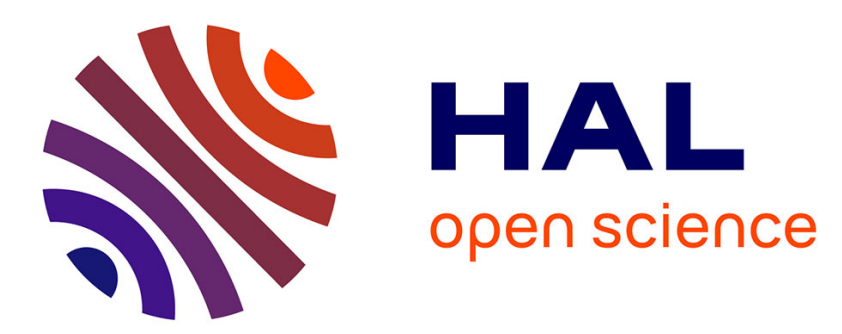

\title{
Instability of the vertical annular flow with a radial heating and rotating inner cylinder
}

\author{
Harunori N. Yoshikawa, Masato Nagata, Innocent Mutabazi
}

\section{To cite this version:}

Harunori N. Yoshikawa, Masato Nagata, Innocent Mutabazi. Instability of the vertical annular flow with a radial heating and rotating inner cylinder. Physics of Fluids, 2013, 25, pp.114104. hal00904851v2

\section{HAL Id: hal-00904851 \\ https://hal.science/hal-00904851v2}

Submitted on 20 Nov 2013

HAL is a multi-disciplinary open access archive for the deposit and dissemination of scientific research documents, whether they are published or not. The documents may come from teaching and research institutions in France or abroad, or from public or private research centers.
L'archive ouverte pluridisciplinaire HAL, est destinée au dépôt et à la diffusion de documents scientifiques de niveau recherche, publiés ou non, émanant des établissements d'enseignement et de recherche français ou étrangers, des laboratoires publics ou privés. 
AIP/123-QED

Instability of the vertical annular flow with a radial heating and rotating inner cylinder

H. N. Yoshikawa, ${ }^{1, \text { a) }}$ M. Nagata, ${ }^{1, \text { b) }}$ and I. Mutabazi ${ }^{1, c)}$

Laboratoire Ondes et Milieux Complexes, UMR 6294 CNRS - Université du Havre

53, rue de Prony - 76058 Le Havre Cedex, France

(Dated: 20 November 2013)

A linear stability analysis of the flow confined in a differentially rotating cylindrical annulus with a radial temperature gradient has been performed. Depending on values of control parameters (the Taylor number, the Grashof number and the Froude number), it has shown flow destabilization to axisymmetric or non axisymmetric modes. Analysis of different terms involved in the evolution rate of the perturbation kinetic energy has allowed us to isolate the dominant terms (centrifugal force or buoyancy force) in the destabilization process. We have shown that the centrifugal buoyancy can induce the asymmetry of the temperature gradient on critical states.

a) Present address: Laboratoire J.-A. Dieudonnée, UMR 7351 CNRS, Université de Nice-Sophia Antipolis, 06108 Nice Cedex 02, France

b) Also at Departement of Aeronautical Engineering, Kyoto University, Japan.

c)Electronic mail: innocent.mutabazi@univ-lehavre.fr 


\section{INTRODUCTION}

In many mechanical systems, there is a need to remove heat through fluid between stationary and rotating elements ${ }^{1-3}$. The purpose of the present investigation is to explore the condition under which vortices can appear in a flow between two coaxial vertical cylinders with a radial temperature gradient and with rotating the inner cylinder. This problem has been investigated both experimentally and numerically, leading to controversial results. In their experimental study with a large radius ratio system, Snyder \& Karlsson ${ }^{4}$ have found that small temperature gradients stabilize the flow, while large values destabilize it. In the destabilized region, vortices are uneven spirals with an almost constant wavelength and an azimuthal wavenumber that varies with temperature gradient. The flow pattern has no axial motion but it moves along the azimuth with an angular velocity almost a half of the inner cylinder velocity. Sorour \& Coney $^{5}$ performed experiments with two values of radius ratio and two different highly viscous oils. They found a continuous destabilization effect of the temperature gradient and observed that critical modes appeared in form of toroidal vortices of an uneven size, traveling along the azimuth but without axial motion. It should be mentioned that both experiments of Snyder et al. ${ }^{4}$ and Sorour et al. ${ }^{5}$ have plotted the critical value of the Taylor number against the temperature gradient but not against any dimensionless control parameter such as Grashof or Rayleigh number.

Most of theoretical studies performed on this problem have made drastic assumptions such as negligible Archimedean buoyancy ${ }^{6,7}$ or axisymmetric perturbations ${ }^{8}$ so that they could not explain experimental results in the vertical annulus. Using flow visualization, Ball \& Farouk ${ }^{3,9}$ have studied the effects of buoyancy on circular Couette air flow in a cylindrical annulus with three different values of the radius ratio. They found that the Taylor vortices become distorted before transition to a spiral vortex flow as the buoyancy is increased. They classified flow regimes using the mixed convection parameter $\mathrm{Ri}$, which is the ratio of the buoyancy to inertia forces and is identical to the Richardson number: For $\mathrm{Ri}<0.01$, the flow pattern is almost the Taylor-Couette pattern as the rotation is dominant over the buoyancy; for $\mathrm{Ri} \in[0.01,10]$, rotation and buoyancy forces affect the flow properties and for $\mathrm{Ri}>10$, only the buoyancy force has a strong influence in the flow destabilization. A thorough linear stability analysis has been performed by Ali \& Weidman ${ }^{10}$. These authors included in their analysis the Archimedean buoyancy and tested stability to both axisymmetric and 
non-axisymmetric perturbations varying the radius ratio and the Prantdl number. They found that the stabilization by the temperature gradient depended both on the radius ratio and the Prantdl number of the working fluids. This study discussed in detail the discrepancies between their theoretical results and experimental results, especially the range of stabilization. They have also developed the arguments of symmetries that should exhibit flow pattern observed in experiments. However, this study has neglected the centrifugal buoyancy.

In a wide-gap experiment, Lepiller et $a l .{ }^{11}$ have shown that the radial temperature gradient is destabilizing independently of its sign, the critical modes appear in the form of a helicoidal vortex flow and the vortex size increases with the radial temperature gradient to a certain value, beyond which the pattern becomes insensitive to the increase of the radial temperature gradient. Motivated by these experimental results, we have revisited the linear stability analysis for this experimental configuration in order to explain the origin of the observed vortical structures. We have formulated the flow problem with minimum assumptions compared to previous studies, i.e., with only adopting the Boussinesq approximation. We have retained both Archimedean and centrifugal buoyancies in our study and we have tested the stability against both axisymmetric and non-axisymmetric perturbations.

The paper is organized as follows: in the next section, we present the flow equations, including base flow solution and linearized equations of perturbations. In Sec. III, we address the inviscid limit of the linearized equations. The results from the linear stability analysis are presented in Sec. IV. Section V contains discussion and the last section addresses concluding remarks.

\section{FLOW EQUATIONS}

We consider a Newtonian fluid (with the density $\rho$, the viscosity $\nu$, the thermal diffusivity $\kappa$ and the thermal expansion coefficient $\alpha$ ) in the annular gap between two coaxial cylinders of infinite length. The inner and outer cylinders of radii $R_{1}$ and $R_{2}\left(=R_{1}+d\right)$ are maintained at temperatures $T_{1}$ and $T_{2}$, respectively. The inner cylinder rotates with the angular frequency $\Omega_{1}$, while the outer one is at rest. The fluid in the annular gap then pertains to a temperature gradient, which is assumed small so that the Boussinesq approximation remains valid. The density will vary with the temperature only in the Archimedean 
and centrifugal buoyancy terms. ${ }^{11}$ The equations of conservation of mass, momentum, and energy read in the cylindrical coordinates $(r, \varphi, z)$ :

$$
\begin{aligned}
& \frac{1}{r} \frac{\partial(r u)}{\partial r}+\frac{1}{r} \frac{\partial v}{\partial \varphi}+\frac{\partial w}{\partial z}=0 \\
& \frac{\partial u}{\partial t}+\left[u \frac{\partial u}{\partial r}+\frac{v}{r} \frac{\partial u}{\partial \varphi}+w \frac{\partial u}{\partial z}-\left(1-G r F r^{2} \theta\right) \frac{v^{2}}{r}\right]=-\frac{\partial p}{\partial r}+\frac{1}{\operatorname{Re}}\left[\left(\triangle-\frac{1}{r^{2}}\right) u-\frac{2}{r^{2}} \frac{\partial v}{\partial \varphi}\right] \\
& \frac{\partial v}{\partial t}+\left[u \frac{\partial v}{\partial r}+\frac{v}{r} \frac{\partial v}{\partial \varphi}+w \frac{\partial v}{\partial z}+\frac{u v}{r}\right]=-\frac{1}{r} \frac{\partial p}{\partial \varphi}+\frac{1}{\operatorname{Re}}\left[\left(\triangle-\frac{1}{r^{2}}\right) v+\frac{2}{r^{2}} \frac{\partial u}{\partial \varphi}\right] \\
& \frac{\partial w}{\partial t}+\left[u \frac{\partial w}{\partial r}+\frac{v}{r} \frac{\partial w}{\partial \varphi}+w \frac{\partial w}{\partial z}\right]=-\frac{\partial p}{\partial z}+\frac{1}{\operatorname{Re}} \Delta w+\frac{G r}{\operatorname{Re}^{2}} \theta \\
& \frac{\partial \theta}{\partial t}+\left[u \frac{\partial \theta}{\partial r}+\frac{v}{r} \frac{\partial \theta}{\partial \varphi}+w \frac{\partial \theta}{\partial z}\right]=\frac{1}{\operatorname{Pr} \operatorname{Re}} \triangle \theta
\end{aligned}
$$

where we have introduced the following scaling to make the flow equations dimensionless: the gap size $d$ for length, $R_{1} \Omega_{1}$ for velocity, $d / R_{1} \Omega_{1}$ for time, $\rho\left(R_{1} \Omega_{1}\right)^{2}$ for pressure and the temperature difference $\Delta \theta=T_{1}-T_{2}$ for temperature. The radial, azimuthal, and axial velocity components are denoted by $u, v$, and $w$, respectively. The temperature $\theta$ is measured with taking $T_{2}$ as reference: $\theta=T-T_{2}$. The Laplacian operator in the cylindrical coordinates is given by

$$
\triangle=\frac{\partial^{2}}{\partial r^{2}}+\frac{1}{r} \frac{\partial}{\partial r}+\frac{1}{r^{2}} \frac{\partial^{2}}{\partial \varphi^{2}}+\frac{\partial^{2}}{\partial z^{2}}
$$

The velocity and temperature fields must satisfy the boundary conditions at cylinder surfaces:

$$
(u, v, w ; \theta)=(0,1,0 ; 1) \text { at } r=\frac{\eta}{1-\eta}, \quad(u, v, w ; \theta)=(0,0,0 ; 0) \text { at } r=\frac{1}{1-\eta}
$$

In Eqs. (1)-(5), we have introduced the following control parameters: the radius ratio $\eta$, the Prandtl number Pr, the Reynolds number Re, the Grashof number $G r$ and the Froude number $F r$ :

$$
\eta=\frac{R_{1}}{R_{2}}, \quad \operatorname{Pr}=\frac{\nu}{\kappa}, \quad \operatorname{Re}=\frac{R_{1} \Omega_{1} d}{\nu}, \quad G r=\frac{\alpha \Delta \theta g d^{3}}{\nu^{2}}, \quad F r=\frac{\nu}{\sqrt{g d^{3}}}
$$

The parameter $\operatorname{GrFr}{ }^{2}=\alpha \Delta \theta$ acounts for the centrifugal buoyancy. This term was neglected in the most previous studies ${ }^{10,12}$ except in the work by Kedia et al. ${ }^{13}$. The prefactor 
Gr $/ \operatorname{Re}^{2}$ of the Archimedean buoyancy in Eq. (4) is identical to the Richardson number Ri, representing the ratio of the buoyancy to inertia forces.

\section{A. Base flow state}

At a small rate of cylinder rotation and a small temperature gradient, the flow is invariant to translation in time (stationarity), to translation along the cylinder axis and to rotation. The base flow state has two velocity components which are functions of the sole radial coordinate $r$ : the azimuthal component $v_{b}$ induced by inner cylinder rotation and the axial component $w_{b}$ induced by the temperature gradient. These velocity components and the temperature field $\theta_{b}$ satisfy the following equations:

$$
\begin{aligned}
& \left(1-G r F r^{2}\right) \frac{v_{b}^{2}}{r}=\frac{\partial p_{b}}{\partial r} \\
& \left(D^{2}+\frac{1}{r} D-\frac{1}{r^{2}}\right) v_{b}=0 \\
& -\operatorname{Re} \frac{\partial p_{b}}{\partial z}+\left(D^{2}+\frac{1}{r} D\right) w_{b}+\frac{G r}{\operatorname{Re}} \theta_{b}=0 \\
& \left(D^{2}+\frac{1}{r} D\right) \theta_{b}=0
\end{aligned}
$$

where $D=d / d r$.

The base state has hence the velocity and temperature profiles given by ${ }^{10,11}$

$$
\begin{aligned}
v_{b} & =\frac{\eta}{1-\eta^{2}}\left[\frac{1}{(1-\eta) r}-(1-\eta) r\right] \\
w_{b} & =\frac{G r}{\operatorname{Re}}\left(C\left[(1-\eta)^{2} r^{2}-1+(1-\eta)^{2} \theta_{b}\right]-\frac{r^{2}(1-\eta)^{2}-\eta^{2}}{4(1-\eta)^{2}} \theta_{b}\right) \\
\theta_{b} & =\frac{\log [(1-\eta) r]}{\log \eta}
\end{aligned}
$$

where the coefficient $C$ is

$$
C=\frac{\left(1-\eta^{2}\right)\left(1-3 \eta^{2}\right)-4 \eta^{4} \log \eta}{16(1-\eta)^{2}\left[\left(1-\eta^{2}\right)^{2}+\left(1-\eta^{4}\right) \log \eta\right]}
$$

The axial velocity component is ascendant near the hot cylindrical surface and descending near the cold one. The base flow has an azimuthal baroclinic vorticity due to temperature 
gradient as well as an axial vorticity due to inner cylinder rotation. The total vorticity of the base flow is

$$
\boldsymbol{\zeta}=-D w_{b} \boldsymbol{e}_{\varphi}+\frac{1}{r} D\left(r^{2} \Omega_{b}\right) \boldsymbol{e}_{z}
$$

where $\Omega_{b}$ is the angular velocity of the flow: $\Omega_{b}(r)=v_{b} / r$. The pressure distribution can be determined by direct integration of Eq. (9), and one will see that radial heating modifies the flow pressure.

\section{B. Stability analysis}

We superimpose infinitesimal perturbations $\left(u^{\prime}, v^{\prime}, w^{\prime}, p^{\prime}, \theta^{\prime}\right)$ on the base flow state. Substitution of the perturbation fields into the flow equations, linearization about the base state and expansion of the perturbations into normal modes

$$
\left(u^{\prime}, v^{\prime}, w^{\prime}, p^{\prime}, \theta^{\prime}\right)=(U, V, W, \Pi, \Theta) e^{s t+i(k z+n \varphi)} .
$$

give rise to the following set of equations:

$$
\begin{aligned}
& \frac{1}{r} D(r U)+\frac{i n}{r} V+i k W=0 \\
& \frac{1}{\operatorname{Re}}\left(\triangle_{k, n}-\frac{1}{r^{2}}\right) U+\left[-\frac{1}{\operatorname{Re}} \frac{i 2 n}{r^{2}}+\frac{2 v_{b}\left(1-G r F r^{2} \theta_{b}\right)}{r}\right] V-D \Pi-G r F r^{2} \frac{v_{b}^{2}}{r} \Theta=(s+i \varpi) U \\
& {\left[\frac{1}{\operatorname{Re}} \frac{i 2 n}{r^{2}}-\left(D v_{b}+\frac{v_{b}}{r}\right)\right] U+\frac{1}{\operatorname{Re}}\left(\triangle_{k, n}-\frac{1}{r^{2}}\right) V-\frac{i n}{r} \Pi=(s+i \varpi) V} \\
& -D w_{b} U+\frac{1}{\operatorname{Re}} \triangle_{k, n} W-i k \Pi+\frac{G r}{\operatorname{Re}^{2}} \Theta=(s+i \varpi) W \\
& -D \theta_{b} U+\frac{1}{\operatorname{Pr} \operatorname{Re}} \triangle_{k, n} \Theta=(s+i \varpi) \Theta
\end{aligned}
$$

where $s=\sigma+i \omega$ is the complex growth rate, $n$ is the azimuthal mode number and $k$ is the axial wavenumber. For brevity, we have set

$$
\varpi=n \Omega_{b}+k w_{b}, \quad \triangle_{k, n}=D^{2}+\frac{1}{r} D-\frac{n^{2}}{r^{2}}-k^{2}
$$


Ali \& Weidman ${ }^{10}$ have shown that the Couette-Taylor flow with a radial temperature gradient, but without centrifugal buoyancy, possesses the following symmetries: If $(U, V, W, \Pi, \Theta ; \operatorname{Re}, G r, k, s, n)$ is a solution of the set of equations (19)-(23),

- then $(U,-V, W, \Pi, \Theta ; \operatorname{Re}, G r, k, s,-n)$ is also a solution which corresponds to the inner cylinder counter-rotating at the same velocity; this symmetry will be denoted by $S^{C / r}\left(\Omega_{b}\right)$;

- then $\left(-U^{*}, V^{*}, W^{*}, \Pi, \Theta ; \operatorname{Re},-G r, k, s^{*},-n\right)$ is also a solution for the same rotation of the inner cylinder, this symmetry will be denoted by $S^{C / r}(\Delta \theta)$.

The presence of the centrifugal buoyancy term in Eq. (20) breaks these symmetries. In the present study we will precise the conditions under which this term can be neglected.

The experimental state diagram of Snyder \& Karlsson ${ }^{4}$ does not exhibit the symmetry $S^{C / r}(\Delta \theta)$, while that of Lepiller et $a l .{ }^{11}$ is relatively symmetric. Snyder \& Karlsson attributed the lack of the symmetry to the Rayleigh instability in the centrifugal field. The present study attempts to explain the origin of this asymmetry.

\section{INVISCID THEORY}

The system of linear stability equations can be truncated in order to investigate the stability criterion in the inviscid limit. This analysis is valid in almost infinite medium in the radial direction (i.e. no-slip boundary conditions cannot be imposed at the cylindrical surfaces). The resulting system can be written in the form ${ }^{14,15}$ :

$$
s \Psi+M \Psi=-\nabla P .
$$

The inertial operator $\boldsymbol{M}$ and the vectors $\boldsymbol{\Psi}$ and $\boldsymbol{\nabla} P$ are given by

$$
\boldsymbol{M}=\left(\begin{array}{cccc}
i \varpi & -\frac{2 v_{b}}{r}\left(1-A \theta_{b}\right) & 0 & A \frac{v_{b}^{2}}{r} \\
D v_{b}+\frac{v_{b}}{r} & i \varpi & 0 & 0 \\
D w_{b} & 0 & i \varpi & -\mathrm{Ri} \\
D \theta_{b} & 0 & 0 & i \varpi
\end{array}\right), \quad \boldsymbol{\Psi}=\left(\begin{array}{c}
U \\
V \\
W \\
\Theta
\end{array}\right), \quad \nabla P=\left(\begin{array}{c}
D P \\
i n P / r \\
i k P \\
0
\end{array}\right)
$$


where $A=\operatorname{Gr} \operatorname{Fr}^{2}(=\alpha \Delta \theta)$. According to Bayly ${ }^{14}$, the eigenvalues of the inertial operator allow to predict the necessary condition for flow instability. We found, as the eigenvalues of $\boldsymbol{M}$,

$$
s_{1,2}=i \varpi \pm \sqrt{-\Phi}, \quad s_{3,4}=i \varpi
$$

where

$$
\Phi(r)=\Phi_{\text {cent }}(r)+\Phi_{t h}(r)
$$

is the generalized Rayleigh discriminant. The ordinary centrifugal Rayleigh discriminant $\Phi_{\text {cent }}$ is given by ${ }^{16,17}$

$$
\Phi_{\text {cent }}=\frac{1}{2 r^{3}} D\left(r^{4} \Omega_{b}^{2}\right)
$$

and the centrifugal buoyancy contribution to the discriminant $\Phi_{t h}$ is given by

$$
\Phi_{t h}=-A\left(\theta_{b} \Phi_{c e n t}+r \Omega_{b}^{2} D \theta_{b}\right)
$$

The same expression shall be obtained by the application of the so-called displaced particle $\operatorname{argument}^{15,18}: \Phi$ is proportional to the density of restoring force acting on displaced particle from its equilibrium position. The term $\Phi_{t h}$ induces an asymmetry between heating the inner cylinder and the outer cylinder. In fact, if $A<0$ (i.e., the inner cylinder is cooler than the outer one), the centrifugal instability is reinforced, while it is weakened if $A>0$ (the outer cylinder cooler than the inner one). This result is in a good agreement with the result of $\mathrm{Yih}^{19}$ on revolving fluids with variable density. The inviscid analysis shows that the centrifugal buoyancy breaks the symmetries $S^{C / r}\left(\Omega_{b}\right)$ and $S^{C / r}(\Delta \theta)$.

Assuming that the operator $\boldsymbol{M}$ is diagonalizable, ${ }^{14}$ we have found an eigenbasis $\left\{\boldsymbol{e}_{i}\right\}$ $(i=1,2,3,4)$ and its adjoint $\left\{\boldsymbol{e}_{i}^{+}\right\}$in which the vector $\boldsymbol{\Psi}$ is represented by the components $\left\{\tilde{\Psi}_{i}\right\}\left(=\boldsymbol{\Psi} \cdot \boldsymbol{e}_{i}^{+}\right)$:

$$
\begin{aligned}
& \frac{d \tilde{\Psi}_{1}}{d t}+(\sigma+i \varpi) \tilde{\Psi}_{1}=-\frac{1}{2} \frac{d \tilde{P}}{d r}+\frac{i n \Omega_{b} \tilde{P}}{\sigma} \\
& \frac{d \tilde{\Psi}_{2}}{d t}+(-\sigma+i \varpi) \tilde{\Psi}_{2}=-\frac{1}{2} \frac{d \tilde{P}}{d r}-\frac{i n \Omega_{b} \tilde{P}}{\sigma} \\
& \frac{d \tilde{\Psi}_{3}}{d t}+i \varpi \tilde{\Psi}_{3}=i k \tilde{P} \\
& \frac{d \tilde{\Psi}_{4}}{d t}+i \varpi \tilde{\Psi}_{4}=0
\end{aligned}
$$


together with the incompressibility condition

$$
\frac{1}{r} \frac{\partial}{\partial r}\left(r \tilde{\Psi}_{1}+r \tilde{\Psi}_{2}\right)+\frac{\sigma}{2 \Omega_{b} r} \frac{\partial}{\partial \varphi}\left(\tilde{\Psi}_{2}-\tilde{\Psi}_{1}\right)+\frac{\partial \tilde{\Psi}_{3}}{\partial z}=0
$$

where $\sigma=\sqrt{-\Phi}$. The component $\tilde{\Psi}_{4}$ is the temperature perturbation (temperature mode). It oscillates at the frequency $\varpi$ and is decoupled from the hydrodynamic fields $\left(\tilde{\Psi}_{1}, \tilde{\Psi}_{2}, \tilde{\Psi}_{3}\right)$. The eigenvectors $\left\{\boldsymbol{e}_{i}\right\}$ and $\left\{\boldsymbol{e}_{i}^{+}\right\}$are given in the Appendix.

\section{VISCOUS THEORY}

The system of equations (19)-(23) together with the homogenous boundary conditions on cylinder surfaces yield an eigenvalue problem $\mathcal{F}(T a, G r, \operatorname{Pr}, F r, \eta ; q, n, s)=0$, where we have introduced the Taylor number $T a$ and the total wavenumber $q$ defined at the median surface

$$
T a=\operatorname{Re} \sqrt{\frac{1-\eta}{\eta}}, \quad q=\sqrt{k^{2}+k_{\varphi}^{2}}
$$

where $k_{\varphi}$ is the wavenumber in the azimuthal direction: $k_{\varphi}=2 n(1-\eta) /(1+\eta)$. We solved the eigenvalue problem $\mathcal{F}=0$ by a Chebyshev collocation method: the velocity, pressure and temperature perturbations $(U, V, W, \Pi, \Theta)$ were expanded into Chebyshev polynomials. The highest order of the polynomials $N$ was typically set at $N=30$, which is sufficient for the convergence. The set of equations (19)-(23) were then discretized and considered only at the Chebyshev-Gauss-Lobatto collocation points. Completed by the boundary conditions, they yielded a generalized eigenvalue problem in a matrix form. Its eigenvalues and eigenvectors were computed by the QZ-decomposition.

The linear stability analysis has been largely investigated by Ali \& Weidman ${ }^{10}$ without the centrifugal buoyancy term. We have reproduced the same shape of the marginal stability curves. We will focus only on new effects which were not presented in their study. We have concentrated our attention to fixed value of $\operatorname{Pr}=5.5$ and $\eta=0.8$ (Fig. 1) corresponding to the experiments of Lepiller et al. ${ }^{11}$, in which the Froude number is $\mathrm{Fr} \sim 10^{-4}$. We have also computed some critical states corresponding to other experiments for comparison.

For $G r \in[-22.5,22.5]$, critical modes are stationary vortices (Fig. 2a) and their threshold is slightly larger than that of the isothermal case $T a_{c}(G r=0)=47.43$. It means that small temperature gradients stabilize the flow. For $|G r|>22.5$, critical modes have non-zero 


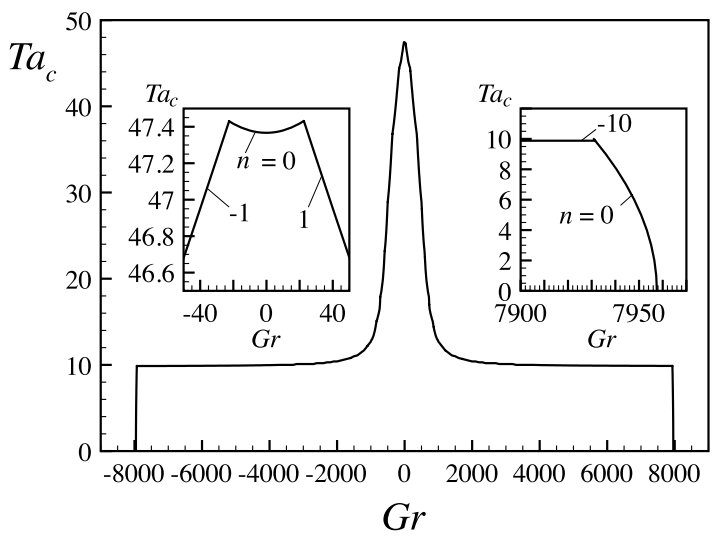

(a)

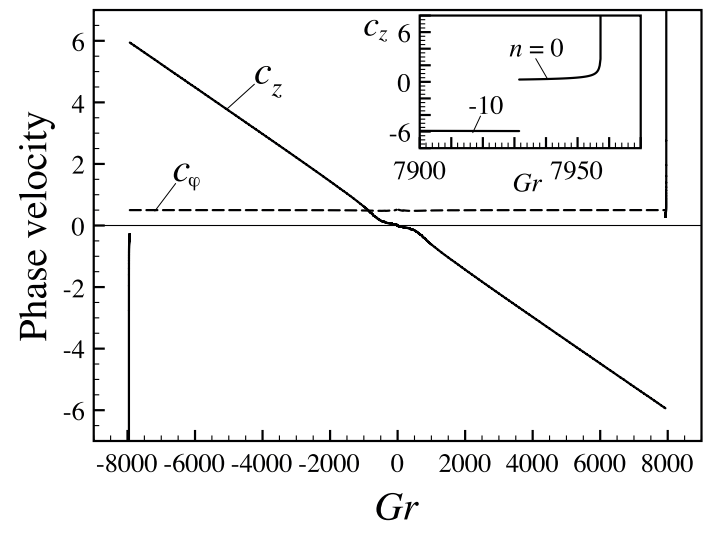

(c)

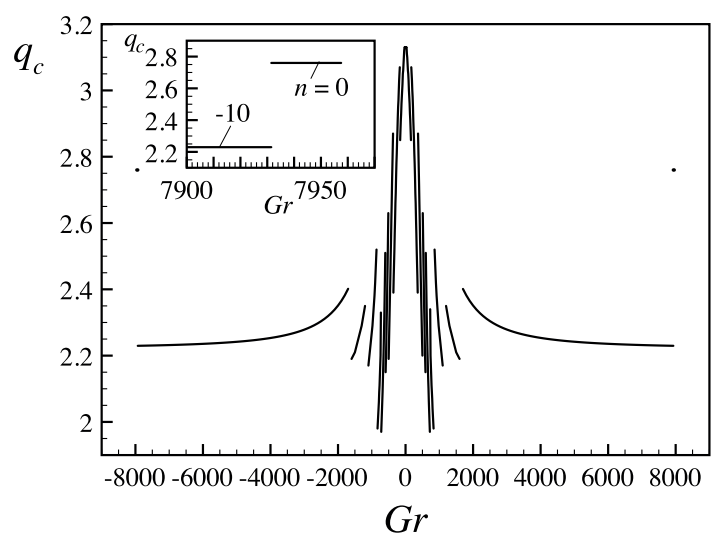

(b)

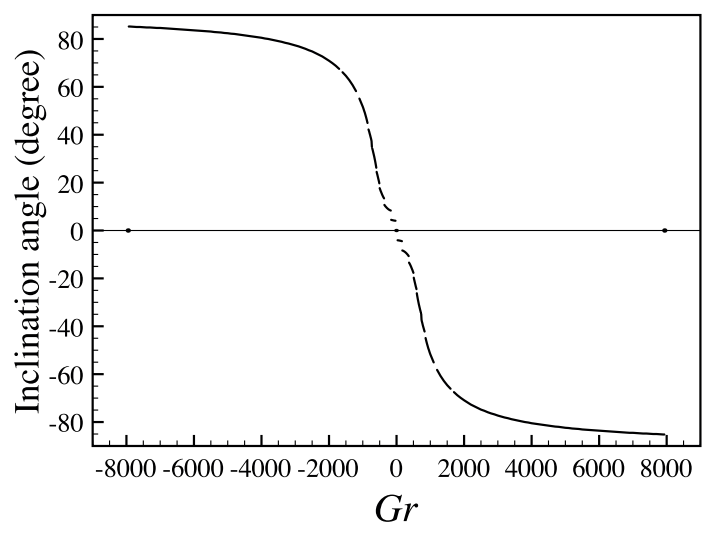

(d)

FIG. 1. Variation of the critical parameters with $G r$ for $\operatorname{Pr}=5.5, \eta=0.8$ and $F r=7.3 \times 10^{-4}$ : (a) $T a_{c}$, (b) $q_{c}$, (c) Phase velocity components and (d) Inclination angle.

frequency and non-zero azimuthal wavenumber $n$.

Therefore these modes are inclined with respect to the cylinder axis and traveling along the azimuth, i.e., the pattern is a spiral vortex flow (Fig. 2bc). The azimuthal wavenumber $n$ counts the number of spirals which are packed around the cylindrical annulus. This number increases with $G r$ but remains smaller than $n_{\max }=\operatorname{int}[\pi(1+\eta) / 2(1-\eta)]=14$ for $\eta=0.8$. The increase of $n$ leads to the increase of the inclination angle $\theta=\tan ^{-1}\left(k_{\varphi} / k\right)$ of the roll and saturates to about $80^{\circ}$. The present flow cannot exhibit vertical rolls (columns). The threshold $T a_{c}$ decreases with $G r$, i.e., the flow is destabilized by the temperature gradient. The critical axial wavenumber decreases also with $G r$, meaning that the radial heating increases the roll size. The total wavenumber of the critical modes decreases with $G r$ from $q_{c}=3.12$ for Taylor vortices to a value around 2 (Fig. 1b). For $|G r|>1500$, there is no significant variation of the critical values of $T a$ and $q$. 


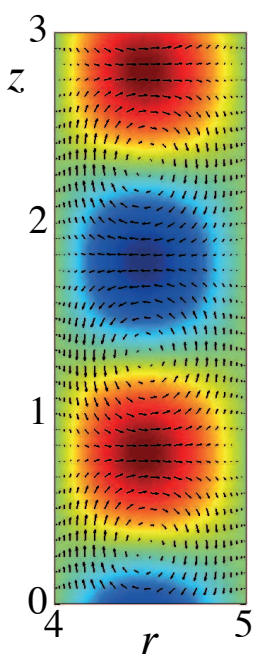

(a) $G r=5$

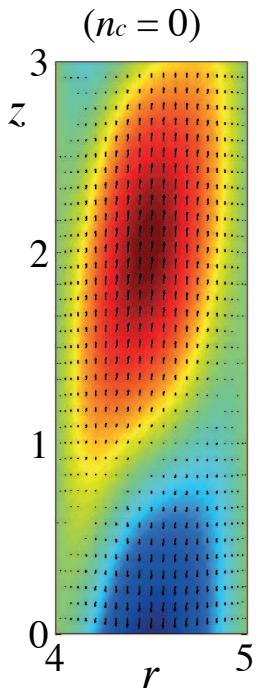

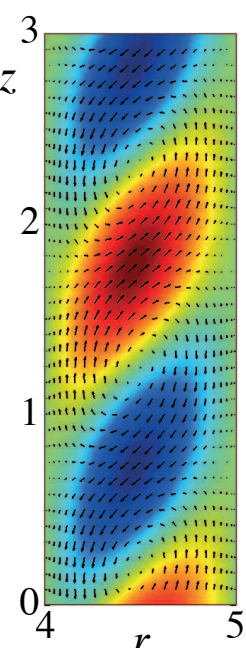

(b) $G r=200$

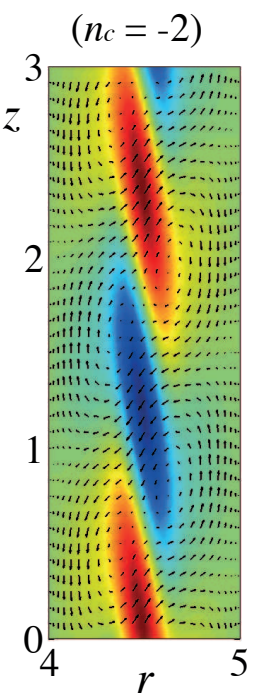

(d) $G r=7958$ $\left(n_{c}=0\right)$
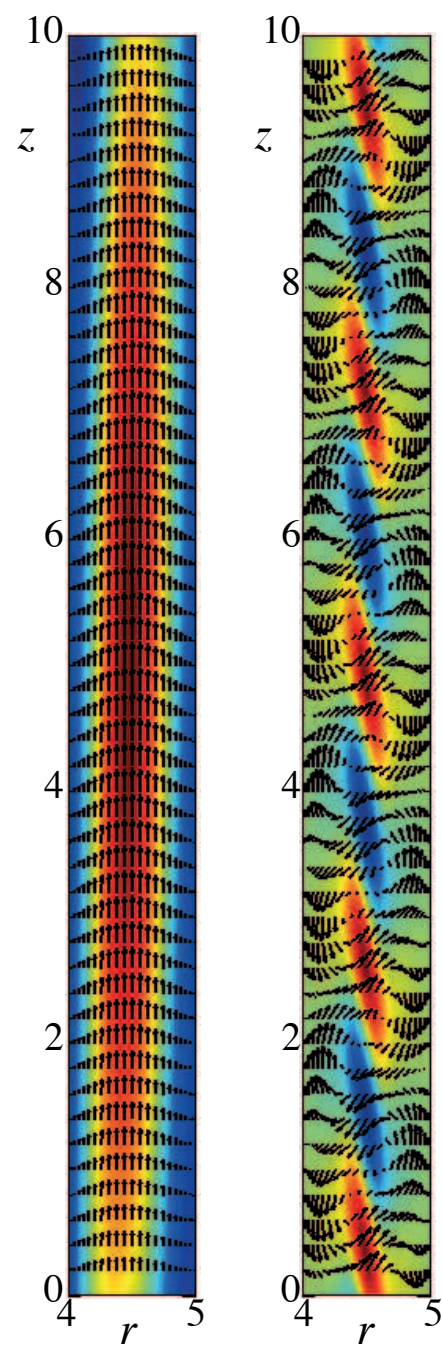

(e) At a codimension-two point $(G r=7932, T a=9.88)$ : $n_{c}=-10$ (left), $n_{c}=0$ (right)

FIG. 2. Critical eigenfunctions for $\eta=0.8, \operatorname{Pr}=5.5$ and $\mathrm{Fr}=7.3 \times 10^{-4}$ : Perturbation velocity and temperature fields are show by arrows and colors, respectively.

For $G r=7932$, there are two marginal curves with the same critical value of $T a_{c} \simeq 10$ but distinct critical values of wavenumbers and frequency. The corresponding point in the plane $\left(G r, T a_{c}\right)$ is called a codimension-two point (Fig. 1a). The coordinates $\left(G r^{++}, T a^{++}\right)$ of the codimension-two points are given in table I. The corresponding modes are shown in Fig. 2e for $G r=7932$. For $G r>7932$, critical modes are stationary and the threshold falls rapidly to $T a_{c}=0$ for $G r=7958$, which corresponds to the onset of natural convection in the case of stationary annulus. ${ }^{20}$

Kinematic description of the critical modes is obtained with the axial phase velocity 
TABLE I. Values of the intersection of the hydrodynamic and thermal modes.

\begin{tabular}{ccc|ccc|ccc}
\hline \hline $\operatorname{Pr}$ & $\eta$ & $F r$ & $T a^{+}$ & $G r^{+}$ & $\mathrm{Ri}^{+}$ & $T a^{++}$ & $G r^{++}$ & $\mathrm{Ri}^{++}$ \\
\hline 0.71 & 0.5 & 0 & 45.3 & 1606 & 0.78 & 28.3 & 8833 & 11.0 \\
5.5 & 0.8 & $7.3 \times 10^{-4}$ & 29.4 & 488.3 & 0.14 & 9.88 & 7932 & 20.3 \\
5.79 & 0.958 & $2.2 \times 10^{-3}$ & 18.6 & 743.7 & 0.094 & 6.86 & 7854 & 7.32 \\
\hline \hline
\end{tabular}

$c_{z}=\omega / k$ and azimuthal phase velocity $c_{\varphi}=\omega / k_{\varphi}$, where $\omega=\operatorname{Im}(s)$. It is found the axial phase velocity $c_{z}$ varies linearly with $G r$ for $|G r| \in[22.5,7932]$, while $c_{\varphi}$ remains constant in the same interval, i.e., it is not modified by the radial temperature gradient. The linear variation of $c_{z}$ with $G r$ was predicted by the inviscid model.

The flow patterns are illustrated in Fig. 2 for different vales of $G r$ by their eigenfunctions. For small values of $G r$, the isotherms are circular lines and the velocity field is similar to that of Taylor vortices. As $G r$ increases, the velocity and temperature fields are distorted and the separatrices are inclined lines with respect to the horizontal direction.

\section{DISCUSSION}

There exist four experiments ${ }^{3-5,11}$ that can be discussed within the present model. Lepiller et $a l .{ }^{11}$ have plotted the critical Taylor numbers in the $G r-T a$ plane from different experiments $^{4,5,11}$. It was found that stabilization occurs even in the experiment ${ }^{5}$. The experiments of Lepiller et al. ${ }^{11}$ were performed with relatively large temperature gradient for which $|G r|>150$, so that they may have missed the stabilization zone. The flow stabilization by weak temperature gradient can be explained as the dominance of the axial velocity of the critical mode compared to the axial velocity induced by the temperature gradient. The present theory predicts the stabilization for weak temperature gradient, i.e., for $|G r|<G r_{s}$ and the destabilization effect observed in the three experiments for $|G r|>G r_{s}$. The value of $G r_{s}$ was discussed in Ali \& Weidman ${ }^{10}$ but its origin remains unclear. In the stabilization zone, critical states are Taylor vortices. In the destabilization zone, critical states appeared in the form of inclined vortices traveling around the cylinder with drift velocity $c_{\varphi}$ that scales with $\left\langle\Omega_{b}\right\rangle^{11}$

$$
\left\langle\Omega_{b}\right\rangle=-\frac{\eta}{1+\eta}\left[1+\frac{\log \eta^{2}}{1-\eta^{2}}\right]
$$


The independence of azimuthal phase velocity with the radial temperature gradient (Fig. 1c) is due to the fact that it is scaled by the average rotation frequency of the inner cylinder. ${ }^{11}$

The present problem is sensitive to boundary conditions. Eagles \& Soundelgekar ${ }^{21}$ have $^{-}$ shown that for mixed thermal boundary conditions (Dirichlet and Neumann conditions at the inner and outer surfaces, respectively), the flow is more unstable to axisymmetric perturbations as the heating is increased.

The analysis of the critical curves in Fig. 1 shows that the variation of the critical parameters with $G r$ exhibits three distinct zones: the stabilization zone, a destabilization zone where the critical parameters strongly decrease with $G r$ and a zone where the critical parameters are almost constant. To get a comprehensive explanation of this behavior, we have performed analysis of the kinetic energy equation of the perturbations.

The variation of the perturbation kinetic energy is governed by

$$
\frac{d K}{d t}=W_{T a}+W_{H y}+W_{\theta}+W_{c B}-D_{\mathrm{v}}
$$

with

$$
\begin{aligned}
& K=\int \frac{1}{2}\left(u^{\prime 2}+v^{\prime 2}+w^{\prime 2}\right) d \mathcal{V}, \quad W_{T a}=-\operatorname{Re} \int\left(D v_{b}-\frac{v_{b}}{r}\right) u^{\prime} v^{\prime} d \mathcal{V}, \\
& W_{H y}=-\operatorname{Re} \int D w_{b} u^{\prime} w^{\prime} d \mathcal{V}, \quad W_{\theta}=\frac{G r}{\operatorname{Re}} \int \theta^{\prime} w^{\prime} d \mathcal{V}, \\
& W_{c B}=-\operatorname{Re} G r F r^{2} \int u^{\prime} \frac{v_{b}}{r}\left(\theta^{\prime} v_{b}+2 \theta_{b} v^{\prime}\right) d \mathcal{V} \quad D_{\mathrm{v}}=\int \Phi_{\mathrm{v}} d \mathcal{V},
\end{aligned}
$$

where $W_{T a}$ represents the power performed by the centrifugal force on perturbation, $W_{H y}$ is the power performed by the Reynolds stress on the baroclinic vorticity, $W_{\theta}$ is the power of the Archimedean buoyancy force, $W_{c B}$ is the power of the centrifugal buoyancy, $D_{\mathrm{v}}$ is the viscous dissipation. The expression of the viscous dissipation function $\Phi_{\mathrm{v}}$ in cylindrical coordinates can be found, e.g., in Bird et al. ${ }^{22}$

There are three terms $\left(W_{T a}, W_{\theta}, W_{H y}\right)$ that always contribute to the increase of the perturbation kinetic energy. The viscous dissipation always dampens the kinetic energy. Two of the three destabilizing terms play a strong influence on the nature of critical mode, as they compete strongly enough depending on the value of $G r$ (Fig. 3). For weak $G r$, the term $W_{T a}$ is the dominant power contribution in the variation of the perturbation kinetic 


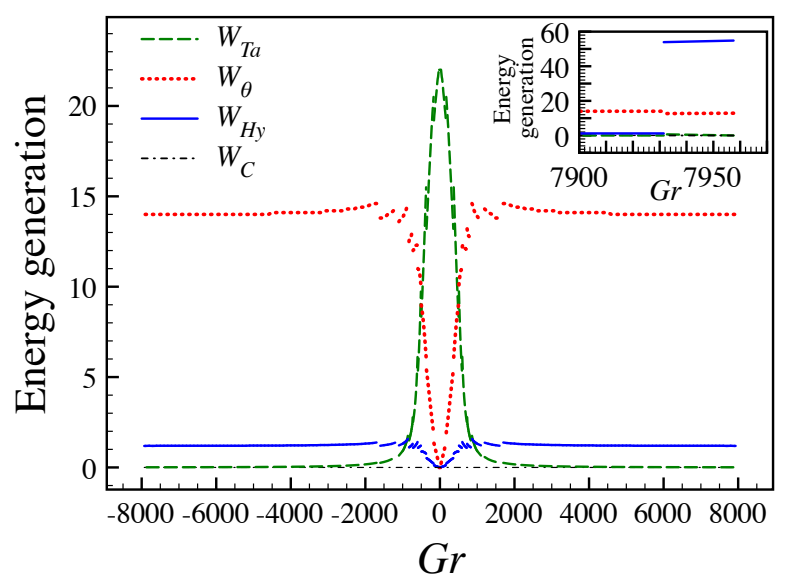

FIG. 3. Variation of different terms contributing to the rate of variation of perturbation kinetic energy $K$ (Eq. 38). The terms have been averaged over a period of oscillations and normalized by $2 K\left(\eta=0.8, \operatorname{Pr}=5.5, F r=7.3 \times 10^{-4}\right)$.

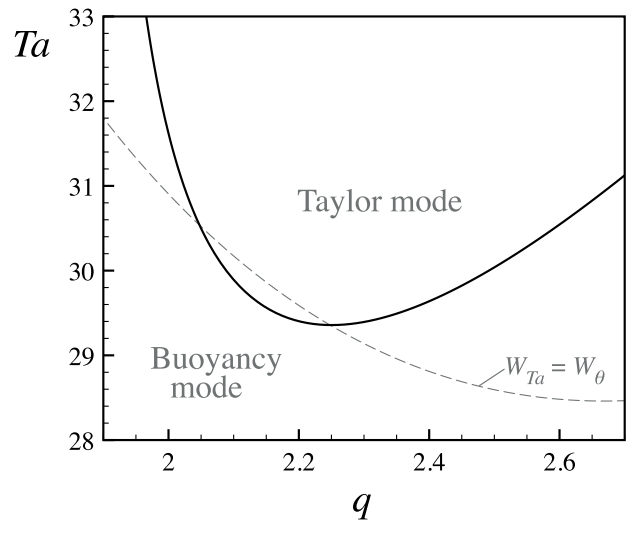

(a) $G r=488.3$

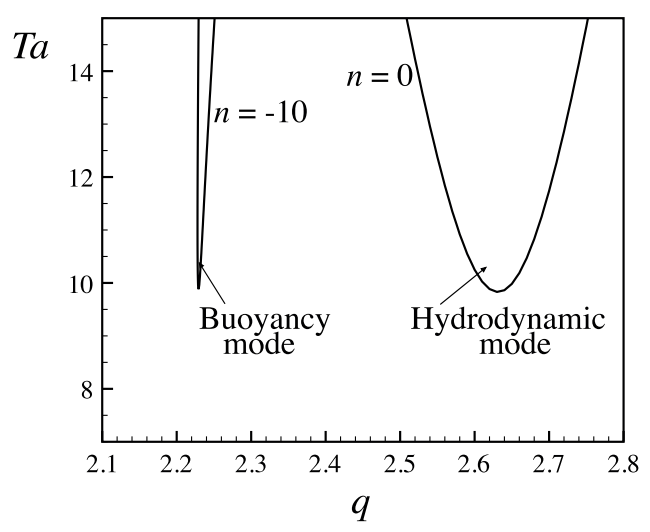

(b) $G r=7932$

FIG. 4. Marginal stability curves of different modes for $\operatorname{Pr}=5.5, \eta=0.8$ and $\mathrm{Fr}=7.3 \times 10^{-4}$.

energy, while for large $G r$, the term $W_{\theta}$ takes over $W_{T a}$ and dominates. In Fig. 4a, we have plotted the marginal stability curve $T a(q)$ for $G r=G r^{+}$where both powers contribute equivalently to the increase of the kinetic energy $K$. In figure, the line of equal centrifugalthermal power, $W_{T a}=W_{\theta}$, is superimposed: the critical state corresponds to the intersection point of the marginal stability curve and the dotted line. The selection of the critical state is therefore determined by the competition between power inputs from centrifugal force and Archimedean buoyancy. The centrifugal buoyancy and the baroclinic vorticity perform weak power for all values of the Grashof number $G r<7932$. Thus, the flow destabilization is mainly due to either the centrifugal force for small $G r$ or Archimedean 
buoyancy for large $G r$. The two mechanisms play an equivalent role for values of $G r=G r^{+}$ and $\mathrm{Ta}=\mathrm{Ta}^{+}$which depend on the $\operatorname{Pr}$ and $\eta$ (table I). For $\eta=0.8$ and $\operatorname{Pr}=5.5$, we found $\mathrm{Gr}^{+}=488.3, \mathrm{Ta}^{+}=29.4$ corresponding to $\mathrm{Ri}^{+}=0.14$. Borrowing the classification of Ball \& Farouk ${ }^{3}$, we can say that for the experimental system used in Lepiller et al. ${ }^{11}$, for $\mathrm{Ri}<0.002$, the centrifugal force dominates and the flow is almost similar to the isothermal case. For $0.002<\mathrm{Ri}<0.1$, the centrifugal force still dominates but the Archimedean buoyancy affects increasingly the flow and modifies its properties. For $0.1<\mathrm{Ri}<3.75$, the centrifugal force decreases, and then for $|G r|>1500$, or $\mathrm{Ri}>3.75$, it has no more effect on the stability. Its power becomes vanishingly small and the Archimedean buoyancy force provides the dominant power in the variation of the perturbation kinetic energy. In this zone, the flow pattern is the result of an excitation of the temperature mode (from the inviscid model) by the rotation. The contribution from the power performed by the Reynolds stress on the baroclinic vorticity remains small until the value of $G r=G r^{++}$where a new instability mode occurs and this contribution pertains a jump to a large value (see inset of Fig. 3). This mode is called hydrodynamic mode and is due to the destabilization of the profile $w_{b}(r)$ that has an inflexion point ${ }^{17}$.

In Fig. 5, we have plotted the normalized values of kinetic energy in the radial, azimuthal and axial directions $(\mathrm{a}-\mathrm{d})$ and the power inputs from different contributions $(\mathrm{e}-\mathrm{h})$. When centrifugal force is dominant, the azimuthal contributions $\left(K_{\varphi}, W_{T a}\right)$ are the most dominant components and when Archimedean buoyancy is dominant, the axial contribution to the kinetic energy $K_{z}$ and the thermal power $W_{\theta}$ are the most important.

The contribution from centrifugal buoyancy can be estimated from the analysis of the terms with the Froude number as a prefactor in Eqs. (20). For this aim, we have computed the critical values of Taylor number as function of $G r$ for five values of the Froude number (Fig. 6a). For small values of Fr, the buoyancy term plays a minor role and the threshold of the instability is almost constant and independent of the sign of $G r$. In this case, the symmetries $S^{C / r}\left(\Omega_{b}\right)$ and $S^{C / r}(\Delta \theta)$ are preserved as investigated by Ali \&Weidman. ${ }^{10}$ This is the case for the experiment of Lepiller et al. ${ }^{11}$ for which $\mathrm{Fr} \sim 10^{-4}$ and where it was shown that the critical values were symmetric with respect to the sign of $G r$. For relatively large values of $F r$, the threshold of the instability depends on the sign of $G r$, the heating of the outer cylinder is more destabilizing than the heating of the inner cylinder. The symmetries $S^{C / r}\left(\Omega_{b}\right)$ and $S^{C / r}(\Delta \theta)$ are broken by the centrifugal buoyancy. This is in agreement 


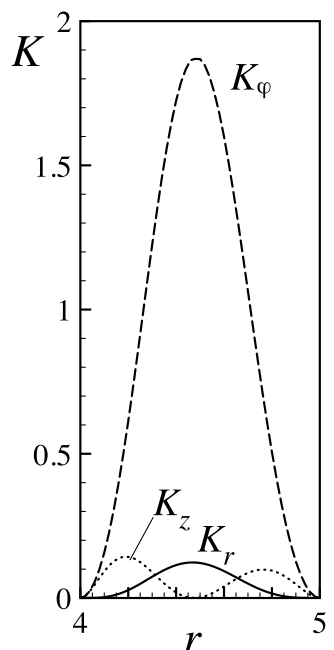

(a) $G r=5$

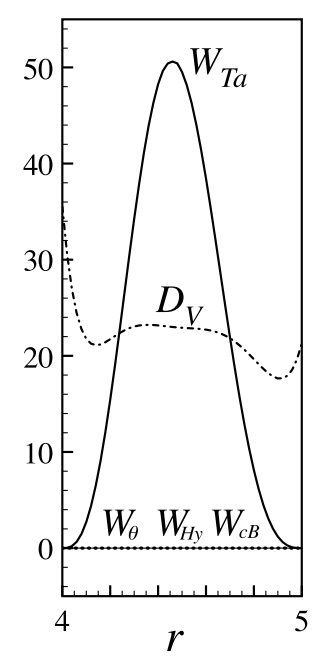

(e) $G r=5$

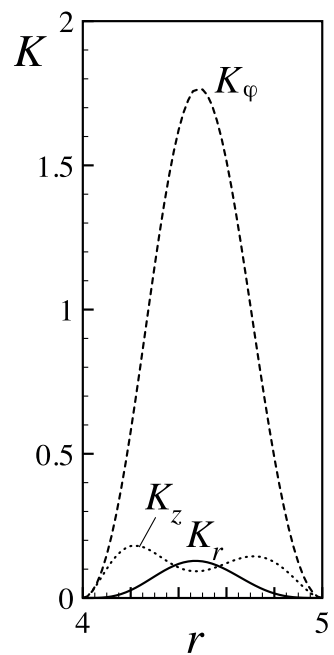

(b) $G r=200$

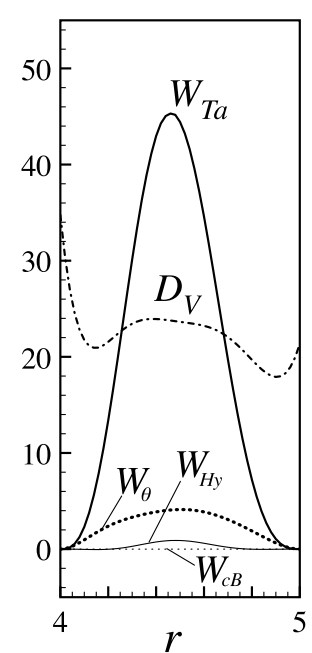

(f) $G r=200$

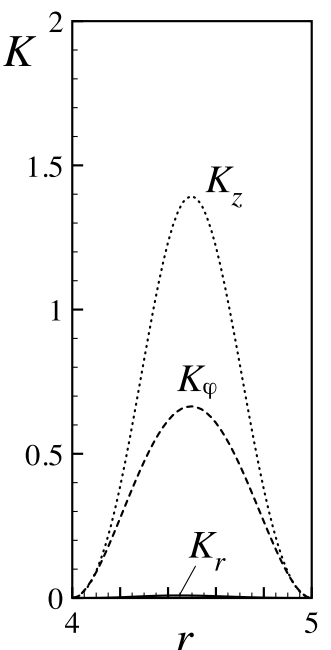

(c) $G r=1000$

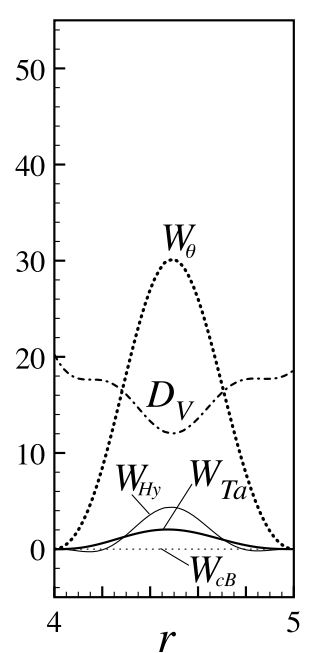

(g) $G r=1000$

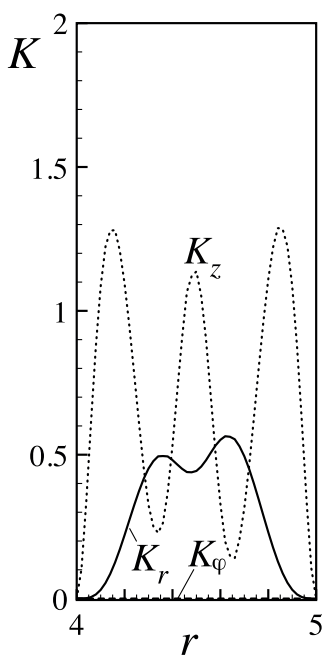

(d) $G r=7958$

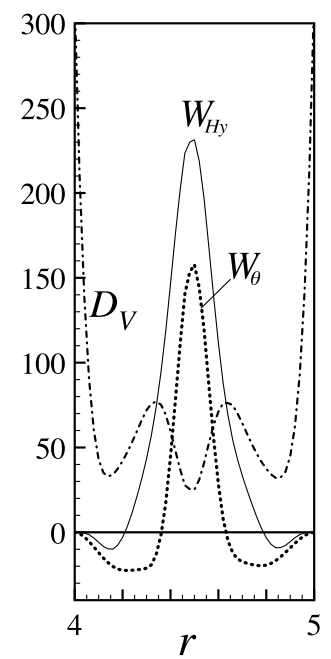

(h) $G r=7958$

FIG. 5. Radial profiles of the different components of the perturbation kinetic energy $K(\mathrm{a}-\mathrm{d})$ and of the different energy generation terms (e-h). $\operatorname{Pr}=5.5, \eta=0.8$ and $\mathrm{Fr}=7.3 \times 10^{-4}$.

with the generalized Rayleigh discriminant of Eqs. (28)-(30). The power performed by the centrifugal buoyancy is negative for $G r>0$, i.e., stabilizing, and positive for $G r<0$, i.e., destabilizing, (Fig. 6b). This is another signature that the centrifugal buoyancy breaks the symmetries $S^{C / r}\left(\Omega_{b}\right)$ and $S^{C / r}(\Delta \theta)$. In most applied situations when the present flow system is encountered (e.g., cooling circuitry of rotating machinery), the gap width is very small and the working fluids can have large values of viscosity, leading to values of $\mathrm{Fr}$ of the order $10^{-2}-10^{-1}$. Therefore the sign of $G r$ has a practical importance on the prediction of the instability threshold. 


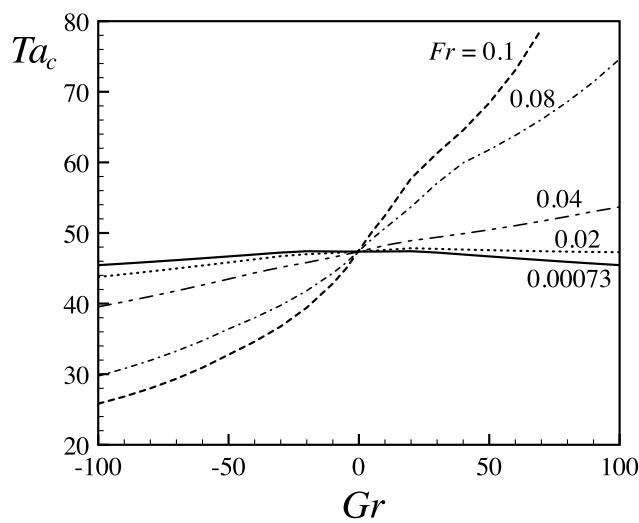

(a)

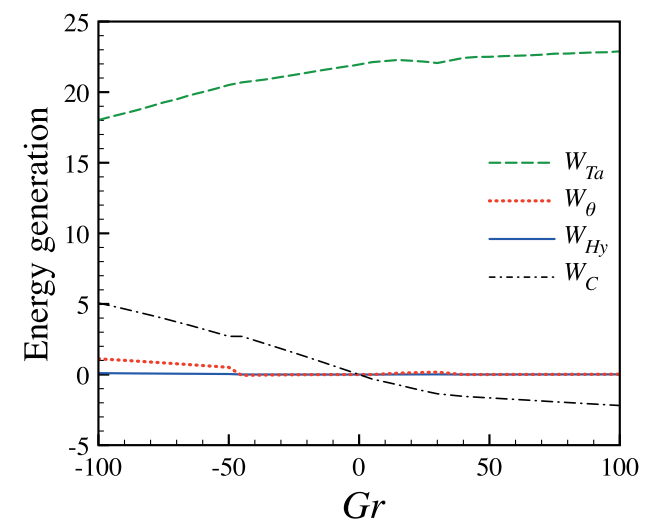

(b)

FIG. 6. Effects of the centrifugal buoyancy for $\eta=0.8$ and $\operatorname{Pr}=5.5$ : (a) Variation of the critical Taylor number $T a_{c}$ with the Grashof number $G r$ for different values of the Froude number $F r$, (b) different energy generation terms for $\mathrm{Fr}=0.08$.

\section{CONCLUSION}

We have performed linear stability analysis of the flow in differentially rotating cylindrical annulus with a radial temperature gradient for fixed values of the Prandtl number Pr and of the radius ratio $\eta$. We have shown that except a small stabilization zone near the isothermal situation, extent of which depends on $\operatorname{Pr}$ and $\eta$, the radial temperature gradient destabilizes the flow and leads to spiral vortices traveling in the azimuth and with large wavelengths compared to the stationary Taylor modes. Energy analysis has allowed us to elucidate the role of each force intervening in the flow: centrifugal force and Archimedean buoyancy interact to generate spiral vortex flow; the centrifugal buoyancy force is responsible for the heating asymmetry. In the codimension-two points, the system can exhibit critical states with different modes. The present analysis has allowed us to have a better understanding of the available experimental results on this flow system. These results call for a detailed numerical simulation to obtain more quantitative data in postcritical regime.

\section{ACKNOWLEDGMENTS}

The present work was issued from various discussions with experimental group in Le Havre (A. Goharzadeh, V. Lepiller, R. Guillerm, C. Savaro and A. Prigent). It has benefited from the financial support from the CPER-Haute Normandie under the program THETE and 
from the FEDER. HNY and IM acknowledge the financial support of the French National Research Agency (ANR), through the program Investissements d'Avenir (ANR-10 LABX09-01), LABEX EMC3. MN was supported by the CNRS FR 3519 (IEPE).

\section{Appendix A: Eigenvectors of the inertial operator $M$}

The direct eigenvectors of the matrix $\boldsymbol{M}$ are:

$$
\boldsymbol{e}_{1}=\left(\begin{array}{c}
1 \\
-\frac{\sigma}{2 \Omega_{b}} \\
D w_{b}-\operatorname{Ri} \frac{D \theta_{b}}{\sigma} \\
\frac{D \theta_{b}}{\sigma}
\end{array}\right), \quad \boldsymbol{e}_{2}=\left(\begin{array}{c}
1 \\
\frac{\sigma}{2 \Omega_{b}} \\
D w_{b}+\operatorname{Ri} \frac{D \theta_{b}}{\sigma} \\
-\frac{D \theta_{b}}{\sigma}
\end{array}\right), \quad \boldsymbol{e}_{3}=\left(\begin{array}{c}
0 \\
0 \\
1 \\
0
\end{array}\right), \quad \boldsymbol{e}_{4}=\left(\begin{array}{c}
0 \\
0 \\
0 \\
-\frac{1}{\mathrm{Ri}}
\end{array}\right)
$$

It has left eigenvectors $\left\{\boldsymbol{e}_{i}^{+}\right\}(i=1,2,3,4)$ are

$$
\boldsymbol{e}_{1}^{+}=\left(\begin{array}{c}
\frac{1}{2} \\
-\frac{\Omega_{b}}{\sigma} \\
0 \\
0
\end{array}\right)^{T}, \quad \boldsymbol{e}_{2}^{+}=\left(\begin{array}{c}
\frac{1}{2} \\
\frac{\Omega_{b}}{\sigma} \\
0 \\
0
\end{array}\right)^{T}, \quad \boldsymbol{e}_{3}^{+}=\left(\begin{array}{c}
0 \\
0 \\
1 \\
0
\end{array}\right)^{T}, \quad \boldsymbol{e}_{4}^{+}=\left(\begin{array}{c}
0 \\
0 \\
0 \\
-\mathrm{Ri}
\end{array}\right)^{T} .
$$

The perturbation vector $\boldsymbol{\Psi}$ can be expressed in the right basis $\left\{\boldsymbol{e}_{i}\right\}(i=1,2,3,4): \mathbf{\Psi}=$ $\sum_{i} \tilde{\Psi}_{i} \boldsymbol{e}_{i}$. The components $\tilde{\Psi}_{i}\left(=\boldsymbol{e}_{i}^{+} \cdot \boldsymbol{\Psi}\right)$ are given by

$$
\left(\begin{array}{c}
\tilde{\Psi}_{1} \\
\tilde{\Psi}_{2} \\
\tilde{\Psi}_{3} \\
\tilde{\Psi}_{4}
\end{array}\right)=\left(\begin{array}{c}
\frac{U}{2}-\Omega_{b} \frac{V}{\sigma} \\
\frac{U}{2}+\Omega_{b} \frac{V}{\sigma} \\
W \\
-\operatorname{Ri} \Theta
\end{array}\right)
$$

In this new basis, $\tilde{\Psi}_{1}$ describes the amplified centrifugal mode with frequency $\varpi ; \tilde{\Psi}_{2}$ is a decaying centrifugal mode; $\tilde{\Psi}_{3}$ is a hydrodynamic wave with frequency $\varpi ; \tilde{\Psi}_{4}=-\operatorname{Ri\Theta }$ describes the temperature wave with frequency $\varpi$. 


\section{REFERENCES}

${ }^{1}$ F. Kreith, "Convection heat transfer in rotating systems," Advances in Heat Transfer 5, 129-251 (1968).

${ }^{2}$ Y. N. Lee and W. J. Minkowycz, "Heat transfer characteristics of the annulus of two coaxial cylinders with one rotating," Int. J. Heat Mass Transfer 32, 711-722 (1989).

${ }^{3}$ B. F. K. S. Ball and V. Dixit, "An experimental study of heat transfer in a vertical annulus with a rotating inner cylinder," Int. J. Heat Mass Transfer 32, 1517-1527 (1989).

${ }^{4}$ H. A. Snyder and S. K. F. Karlsson, "Experiments on the stability of Couette motion with a radial thermal gradient," Phys. Fluids 7, 1696-1706 (1964).

${ }^{5}$ M. M. Sorour and J. E. R.Coney, "The effect of temperature gradient on the stability of flow between vertical, concentric, rotating cylinders," J. Mech. Engng Sci. 21, 403-409 (1979).

${ }^{6}$ J. Walowit, S. Tsao, and R. C. DiPrima, "Stability of flow between arbitrarily spaced concentric cylindrical surfaces including the effect of a radial temperature gradient," ASME J. Appl. Mech. 31, 585-593 (1964).

${ }^{7}$ K. G. Roesner, "Hydrodynamic stability of cylindrical Couette-flow," Arch. Mech. 30, 619-627 (1978).

${ }^{8}$ J.-C. Chen and J.-Y. Kuo, "The linear stability of steady circular Couette flow with a small radial temperature gradient," Phys. Fluids A 2, 1585-1591 (1990).

${ }^{9}$ K. S. Ball and B. Farouk, "Bifurcation and phenomena in Taylor-Couette flow with buoyancy effects," J. Fluid Mech 197, 479-501 (1988).

${ }^{10} \mathrm{M}$. Ali and P. D. Weidman, "On the stability of circular Couette flow with radial heating," J. Fluid Mech. 220, 53-84 (1990).

${ }^{11}$ V. Lepiller, A. Goharzadeh, A. Prigent, and I. Mutabazi, "Weak temperature gradient effect on the stability of the circular Couette flow," Eur. Phys. J. B 61, 445-455 (2008).

${ }^{12}$ K. S. Ball and B. Farouk, "A flow visualization study of the effects of buoyancy on Taylor vortices," Phys. Fluids A 1, 1502-1507 (1989).

${ }^{13}$ M. H. R.Kedia and T. Colonius, "Numerical simulations of heat transfer in Taylor-Couette flow," ASME J. Heat Transfer 120, 65-71 (1998).

${ }^{14} \mathrm{~B}$. J. Bayly, "Three-dimensional centrifugal-type instabilities in inviscid two-dimensional flows," Phys. Fluids 31, 56-64 (1988). 
${ }^{15}$ I. Mutabazi, C. Normand, and J. E. Wesfreid, "Gap size effects on centrifugally and rotationally driven instabilities," Phys. Fluids A 4, 1199-1125 (1992).

${ }^{16} \mathrm{~S}$. Chandrasekhar, Hydrodynamic and hydromagnetic stability (Dover, New York, 1961).

${ }^{17}$ P. G. Drazin and W. H. Reid, Hydrodynamic stability, 2nd ed. (Cambridge University Press, 2004).

${ }^{18}$ L. D. Landau and E. M. Lifshitz, Fluid mechanics, 2nd ed., Landau and Lifshitz Course of Theoretical Physics, Vol. 6 (Elsevier Butterworth-Heinemann, Burlington, Massachusetts, 1989).

${ }^{19}$ C.-S. Yih, "Dual role of viscosity in the instability of revolving fluids of variable density," Phys. Fluids 4, 806-810 (1961).

${ }^{20}$ A. Bahloul, I. Mutabazi, and A. Ambari, "Codimension 2 points in the flow inside a cylindrical annulus with a radial temperature gradient," Eur. Phys. J. AP 9, 253-264 (2000).

${ }^{21}$ V. S. P.M. Eagles, "Stability of flow between two rotating cylinders in the presence of a constant heat flux at the outer cylinder and radial temperature gradient-wide gap," Heat and Mass Transfer 33, 257-260 (1997).

${ }^{22}$ R. B. Bird, W. E. Stewart, and N. Lightfoot, Transport Phenomena, 2nd ed. (John Wiley \& Sons, New York, 1960). 